\title{
STANDARDISED DOCUMENTATION OF INSTRUMENT USE DURING LAPAROSCOPIC SURGERY
}

\author{
M. Zickerow ${ }^{1}$, C. Holmer ${ }^{2}$, M. Kraft ${ }^{1}$ \\ ${ }^{1}$ Fachgebiet Medizintechnik, Technische Universität Berlin, Germany \\ ${ }^{2}$ Chirurgische Klinik I, Charité - Universitätsmedizin Berlin, Germany \\ mzickerow@zmms.tu-berlin.de
}

\begin{abstract}
To date, usability problems for laparoscopic instruments have mainly been investigated using questionnaires or laboratory studies. However, this methodology prevents the duration and frequency of instrument use from being documented objectively. Therefore, a tablet was used to create a graphic user interface which enables instrument use by operating and assisting surgeons to be documented with a high level of temporal precision. In a preliminary study, instrument use was documented for five laparoscopic operations (0:44h-6:01h). 2021 actions and 221 changes of instruments were documented. The longest uninterrupted usage period without changing hands was determined for grasping forceps at $97 \mathrm{~min}$.
\end{abstract}

Keywords: usability, minimally invasive surgery, instrument, usage period

\section{Introduction}

Surgeons' instruments have been developing alongside the discipline itself. As new operations have been developed, so, too, have the instruments needed to perform them, and these are now associated with the names of significant surgeons. Instruments for minimally-invasive interventions hold a special place, as these have only been used more frequently over the past 30 years and often feature a modular construction.

Minimally-invasive operations offer a wide range of advantages for the patient, although they are considered as being more complex and difficult for the surgeon. Besides the frequently-cited fundamental difficulties, such as limitations with regard to the degree of freedom, a lack of haptic feedback and lack of depth information, the instruments themselves are also restrictive.

There are a wide range of surveys [e.g. 2, 4] and studies [e.g. 1,3] that indicate various deficits in terms of usability. One difficulty when it comes to minimising these deficits is that the equipment for an operating theatre is generally not provided by just one manufacturer, but rather various manufacturers are represented with various usage concepts. At the same time, the instruments and, to a higher degree, the devices are multi-functional, although the surgeon's options for using them are severely limited. The surgeon usually holds surgical instruments in both hands, keeps a close eye on his work on a monitor, and operates additional functions using foot or manual switches. If additional settings or functions are required, these must be selected on the device by assistants. The latest developments in integrated operating theatres have positioned operating functions closer to the surgeon via sterile touchscreens, although, as the surgeon tends to use instruments in both hands, these concepts are still rarely used by the surgeon himself. The interaction between the surgeon and technology will therefore remain limited to the use of a few switches and pedals for the foreseeable future. Additional functions can only be used by involving another person, cf. figure 1.

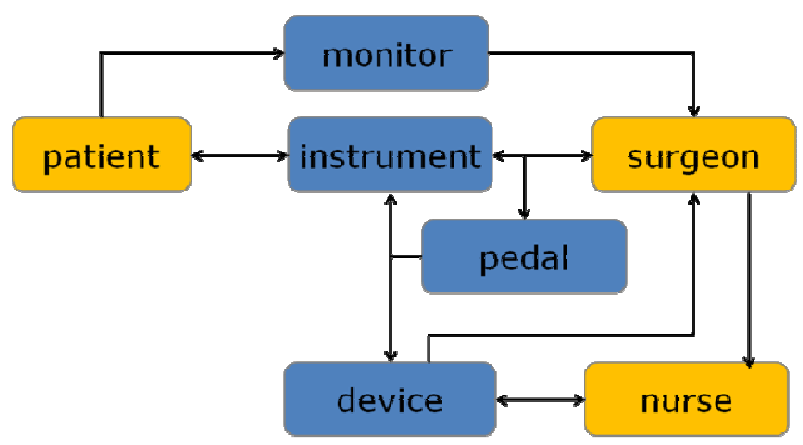

figure 1: surgeon-technology interaction

On-going surgery places limitations on investigation methods, and these limitations pose a problem when it comes to identifying and assessing usability issues. Many of the existing studies therefore use questionnaires or laboratory investigations, which can only replicate reality in a limited manner.

Consequently, von Saucken observed 70 operations and was able to document 400 occurred incidents [4]. Due to the documentation methodology used, it has so far not been possible to make a statement on the likelihood of problems occurring based on the frequency of use. As the first step in this direction, a system was developed and tested which can document the frequency of use and usage period for the instruments used during laparoscopic operations.

\section{Materials}

A tablet (Samsung Ativ Smart PC 500T1C, 11.6 inch) was used to document the course of the operation. The LibreOffice Base software was used to create a graphic user interface (GUI), which enabled the humantechnology interaction to be documented for the operating and an assisting surgeon. In the process, information on the medical context and other people in the operating theatre was intentionally not recorded in order to enable the different actions of the surgeons to be documented with greater temporal and content-related precision. For both surgeons, the instrument used can be selected for 
both hands using a drop-down menu. Depending on the instrument, there are further usage forms available in an additional menu, such as "monopolar coagulation" or "fixing tissue in place". Buttons can be used to record organisational, technical and medical disturbances, delays and other comments.

All interactions entered via the GUI are stored in a database along with a time stamp.

\section{Methods}

To date, five laparoscopic or laparoscopically-supported operations have been documented in Chirurgische Klinik I at Charité - Universitätsmedizin Berlin with an average incision-stitch duration of $2: 15 \mathrm{~h}(0: 44 \mathrm{~h}-6: 01 \mathrm{~h})$. Due to the design of the software interface, only the laparoscopic sections of the operations were recorded.

For each operation, the human-instrument interactions were documented for the operating surgeon $(n=2)$ and the assisting surgeon $(n=3)$.

\section{Results}

To date, 2021 actions have been documented, of which 221 were changes of instrument. The usage period varied considerably (cf. figure 2). In total, an instrument was used 145 times for less than 5 minutes without changing hands, 58 times for longer than 5 but less than 30 minutes, and 18 times for longer than 30 minutes. These long usage intervals mainly occurred for the endoscope grasping forceps, although in two cases for ultrasonic shears (U1tracision (C) Ethicon). The longest usage period so far was documented for grasping forceps at $97 \mathrm{~min}$.
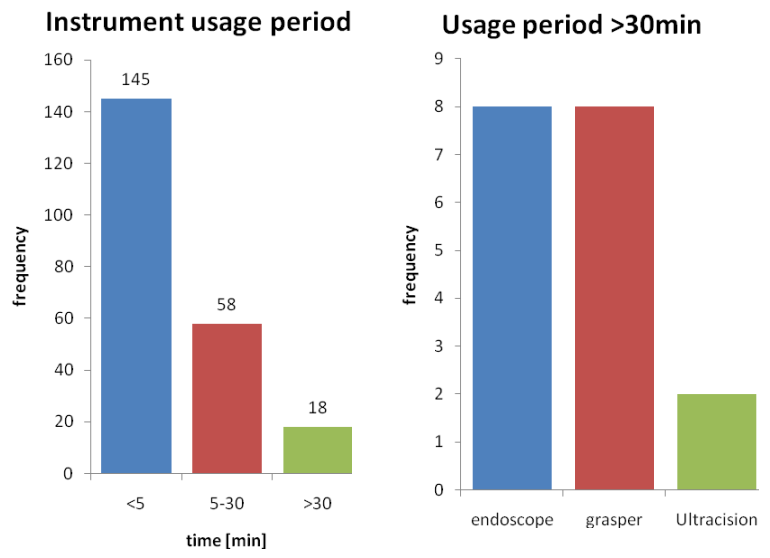

figure 2: instrument usage periods

\section{Discussion}

The previous results show that it is possible to document the actions of surgeons using the software used. The limitation on the interactions of two people was proven, as it was possible to achieve a high level of temporal and content-related precision this way. The evaluation of the usage period for the instruments shows that, in addition to many very fast instrument changes, there are also very long usage phases. The fact that the endoscope and grasp- ing forceps were used for the same amount of time in one hand without changing, although a laparoscopy is impossible without an endoscope, is probably due to the operations observed. There was generally more than one assistant present for these, so the actions of the assistant using the endoscope could not be recorded throughout the documentation period.

Grasping forceps are predominantly not used to separate tissue, but keep the tissue in a certain position in order to prepare the tissue in combination with a further instrument.

In contrast, the Ultracision shears are used mainly to separate tissue with minimal bleeding and for vascular occlusion. The long usage period indicates that a wide range of tasks can be successfully fulfilled with this instrument.

\section{Outlook}

The tested methodology for documenting the course of surgery is now being used for further operations. In addition to purely documenting instrument usage, a larger database potentially also enables statements to be made on problems relating to the usability of the instruments.

\section{Acknowledgments}

We would like to thank Prof. Dr. med. Kreis and his team at Chirurgische Klinik I at Charité - Universitätmedizin Berlin for their friendly assistance.

\section{Bibliography}

[1] Ahmed, S., Hanna G.B., Cuschieri, A.: Optimal Angle Between Instrument Shaft and Handle for Laparoscopic Bowel Suturing, Arch Surg., vol 139, pp. 89-92, 2004

[2] Berguer, R., Hreljac, A.: The relationship between hand size and difficulty using surgical instruments: A survey of 726 laparoscopic surgeons, Surg. Endosc, vol. 18 , pp. 508-512, 2004

[3]Büchel, D., Mårvik, R., et. al.: Ergonomics of disposable handles for minimally invasive surgery, Surg. Endosc., vol 24, pp. 992-1004, 2009

[4] von Saucken, A.-M., Donner, S., Kraft, M.: Ergonomic Problems Originating in the Use of HighFrequency and Ultrasonic Medical Devices, Biomed. Tech., vol. 57 (Suppl. 1), pp. 951-954, 2012 